\title{
The Sex Difference in Hemoglobin Level, Systolic and Diastolic Blood Pressure of Dragon Boat Athletes
}

\author{
Pipit Pitriani*, Mona Fiamentta Febrianty \\ Department of Coaching Education, Faculty of Sports and Health Education \\ Universitas Pendidikan Indonesia \\ Bandung, Indonesia \\ *pipitpitriani@upi.edu, monachalil@gmail.com
}

\begin{abstract}
Dragon boat racing is one of rowing competition. The purpose of this study was to investigate the sex difference of hemoglobin level and blood pressure of dragon boat athletes. This descriptive cross-sectional study with 29-person dragon boat athletes (14-person female and 15-person male). Blood tests are performed to measure hemoglobin levels and blood pressure measurements. The results obtained were significant differences between hemoglobin levels, systolic and diastolic blood pressure in female compared to male. Sex different make the level of hemoglobin and blood pressure different. This finding can be used as a guide for the coach to know the basic characteristics of the athletes.
\end{abstract}

\section{Keywords: blood pressure, dragon boat athletes, hemoglobin}

\section{INTRODUCTION}

Dragon boat racing is a competitive water sport that involves 20 paddlers propelling the boat across race distances ranging from $200 \mathrm{~m}$ to $2,000 \mathrm{~m}$ [1]. Dragon boat racing requires high physical training for the whole body where the athletes push the boat forward with short paddles with horizontal cross cuts at the top of the track [2]. Some of the physiological changes associated with strenuous exercise [3].

Men and women have different average hemoglobin $(\mathrm{Hb})$ levels. Women have an average level of about $12 \%$ lower than men. It is probably a direct effect of sex hormones, both oestrogen and androgens, on erythropoiesis [4]. Hb and Red blood cell (RBC) levels in subjects with resistance training, such as throwing tend to be higher than those with dominant endurance training such as middle and long distance running [5]. Reinke et al. indicated that rowing, in which the most dominant physical factors for performance are power and endurance, alleviates the reduction of iron in comparison to soccer, which is dominated by speed and agility [6].

Beside hemoglobin level, blood pressure is one of the physiological factor that was change by exercise. Shahraki et al., mentioned that physical activity reduced arterial blood pressure (BP) levels in female athlete students [7]. Exercise known can stimulates development in hemodynamic status. When exercise started there will be circulate the blood more to systemic [8].

However, no data explain the different between female and male hemoglobin level and blood pressure in dragon boat athletes. The purpose of this recent study was to investigate the sex different on hemoglobin level and blood pressure in dragon boat athletes.

\section{METHOD}

\section{A. Research Design}

This study used comparative design to investigate the different of hemoglobin level, systolic and diastolic blood pressure between male and female of dragon boat athletes.

\section{B. Subjects}

Twenty-nine dragon boat athletes participated in this study. The participants were divided by sex. Fourteen persons female and fifteen persons male. Written informed consent was obtained from all subjects in addition to completion blood collecting.

\section{Study Procedure}

Blood collected using $3 \mathrm{ml}$ syringe and put in vacutainer with EDTA. The blood diluted with a DCL cell pack. Sulfolyser were added so that the erythrocytes will lysis and the $\mathrm{Hb}$ will be released, the amount of $\mathrm{Hb}$ released is read as hemichromes, read by the SLS hemoglobin in Sysmex XN 550 (Sysmex, Norderstedt, German).

Blood pressure was assessed from the left arm with an ABN Spectrum Aneroid Sphygmomanometer and with $3 \mathrm{~m}^{\mathrm{TM}}$ Littman ${ }^{\circledR}$ Classic III ${ }^{\mathrm{TM}}$ Monitoring Stethoscope. The subject sit in a relaxed position with the arm at rest on a table. Secure the cuff on the bicep and squeeze the balloon to increase the pressure. Watch the aneroid monitor and increase the pressure to about $30 \mathrm{mmHg}$ over the normal blood pressure, or to 180 $\mathrm{mmHg}$ if this is not known. When the cuff is inflated, place the stethoscope just inside the elbow crease under the cuff. Slowly depress the balloon and listen through the stethoscope. The systolic pressure is when the first beats hit, note the number on the aneroid monitor. Continue listening to find the diastolic pressure until the steady heartbeat sound stops and record the number from the aneroid monitor again. Three measurements divided as mean calculated for the blood pressure.

The subject condition when checking blood pressure should follow this condition: take several minutes to 10 minutes rest before check and take a few deep breath, avoid talking during the test, avoid checking blood pressure in a cold room at home, sustenance the arm as close to heart level as possible. Manual cuffs come in different sizes depending on the size of the arm. 


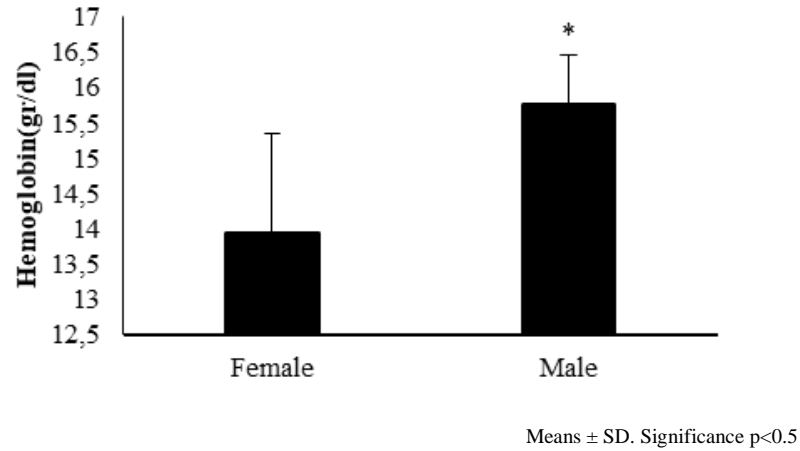

Fig. 2. The hemoglobin difference between female and male.

There was significance different between systolic blood pressure of female and male dragon boat athletes $(\mathrm{p}<0.05)$. The average of female systolic blood pressure was $105 \mathrm{mmHg}$ and $112 \mathrm{mmHg}$ for male systolic blood pressure.

Cardiovascular system has change many physiological part due to exercise such as blood pressure. The level of training can also contribute to the difference in left ventricular mass. Women athletes show smaller left ventricular mass than Men $[11,12]$. A normal response in an apparently healthy subject is a linear increase in SBP as exercise intensity increases. Dimkpa et al., mentioned that young adult males of age 18-35 years showed higher responses in percentage rise of SBP during exercise and higher rate of SBP decline during recovery [13].

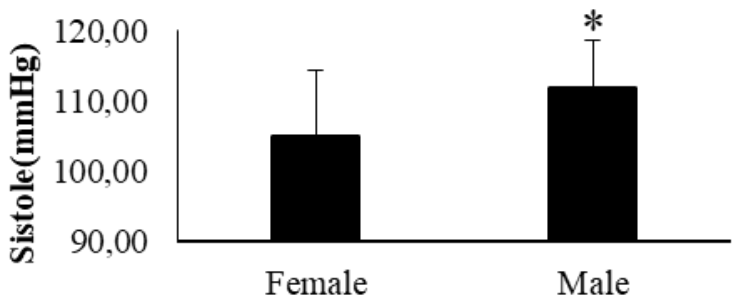

Means \pm SD. *Significance $p<0.5$

Fig. 1. Age difference between female and male.

There was significance difference between hemoglobin level female and male $(\mathrm{p}<0.05)$. The average female hemoglobin level was $13.95 \mathrm{mg} / \mathrm{dL}$ and $15.76 \mathrm{mg} / \mathrm{dL}$ for male. In this recent study found that average hemoglobin level of male was higher than female. Hemoglobin is the component of red blood cells that carries oxygen and carbon dioxide [3].

In healthy humans, the venous hemoglobin level correlates to uncertain amount of red cell mass. The physiological factors may be constitutive or chronic, such as puberty and menopause, acclimatization to altitude, level of fitness or lean body mass, or acute such as posture or level of hydration[4]. Sex differences in mean venous hemoglobin levels and red blood cell mass are related to the direct stimulating effect of androgens in men in the bone marrow with erythropoietin. Effects of androgen stimulation on erythropoietin production in the kidneys, and the use of the hormone estrogen in the female bone marrow[9]. This $\mathrm{Hb}$ mass development was independent from endurance training volume in the age period from 16 to 19 year in male adolescents [10]. 
[3] A. Ghosh and B.N. Kundu, "Effect of exercise on Haemoglobin Percentage among Three Different Physically Active groups," IOSR J. Humanit. Soc. Sci. Ver. IV, vol. 20(5), pp. 34-38, 2015.

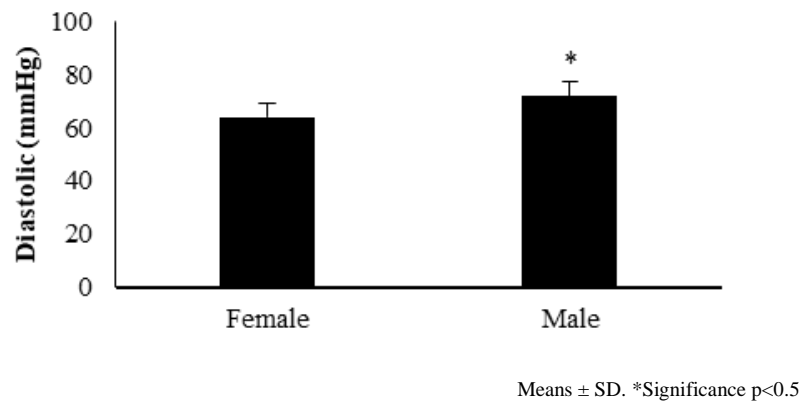

Fig. 4. The diastolic blood pressure difference between female and male.

Forjaz et al., mentioned that the blood pressure change in exercise and recovery period [16]. Diastolic pressure was significantly higher in resting time. The systolic and diastolic blood pressure of male was significantly higher than female. These results was also demonstrated in other study related with the hormonal factor in female that interfere the systolic and diastolic blood pressure [17].

\section{CONCLUSION}

The hemoglobin level, systolic and diastolic blood pressure was significantly different between female and male of dragon boat athletes. The male systolic and diastolic blood pressure was significantly higher than female blood pressure. However, the level of hemoglobin and blood pressure all of the subject was in normal range.

\section{ACKNOWLEDGMENT}

This project funded by the Institute of Research and Community Service Universitas Pendidikan Indonesia.

\section{REFERENCES}

[1] S.R. Ho, R.M. Smith, P.G. Chapman, P.J. Sinclair and K. Funato, "Physiological and physical characteristics of elite dragon boat paddlers," J. Strength Cond. Res, vol. 27(1), pp. 137-145, 2013.

[2] I.A.B. Jun Liang Ong, "Energy Expenditure, Availability, and Dietary Intake Assessment in Competitive Female Dragon Boat Athletes," Sports, vol. 5(4), pp. 45, 2017.
[4] W.G. Murphy, "The sex difference in haemoglobin levels in adults Mechanisms, causes, and consequences," Blood Rev, vol. 28(2), pp. 4147, 2014.

[5] T. Fujii, "Dietary Iron Intake and Hemoglobin Concentration in College Athletes in Different Sports," Int. J. Sport. Exerc. Med, vol. 1(5), pp. 913, 2015.

[6] S. Reinke et al., "Absolute and functional iron deficiency in professional athletes during training and recovery," Int. J. Cardiol, 2012.

[7] M.R. Shahraki, H. Mirshekari, A.R. Shahraki, E. Shahraki and M. Naroi, "Arterial blood pressure in female students before, during and after exercise," ARYA Atheroscler, 2012.

[8] S. Choudhary, Rajnee and B.K. Binawara, "Effect of exercise on serum iron, blood haemoglobin and cardiac efficiency," J. Postgrad. Med. Inst, vol. 26(1), pp. 13-16, 2012.

[9] W. Jelkmann, "Regulation of erythropoietin production," J. Physiol, vol. 589(6), pp. 1251-1258, 2011.

[10] T. Steiner, T. Maier and J.P. Wehrlin, "Effect of Endurance Training on Hemoglobin Mass and V-O 2 max in Male Adolescent Athletes," Med. Sci. Sports Exerc, vol. 51(5), pp. 912-919, 2019.

[11] A.M. Dobosiewicz, "Adaptive mechanisms of cardiovascular system in trained athletes Ilona Malinowska 1, Anna Maria Dobosiewicz 1, Łucja Stalmi rska, Ewelina Pankanin Scientific Circle at Department of Hygiene, Epidemiology and Ergonomics . Division of Ergonomics and Exe,” J. Educ. Heal. Sport, vol. 7(12), pp. 378-385, 2017.

[12] A. žemva and P. Rogel, "Gender differences in athlete's heart: Association with 24-h blood pressure. A study of pairs in sport dancing," Int. J. Cardiol, vol. 77(1), pp. 49-54, 2001.

[13] U. Dimkpa, A. Ugwu and D. Oshi, "Assessment of sex differences in systolic blood pressure responses to exercise in healthy, non-athletic young adults," J. Exerc. Physiol. Online, vol. 11(2), pp. 18-25, 2008.

[14] J.F. Reckelhoff, "Gender differences in the regulation of blood pressure," Hypertension, vol. 37(5), pp. 1199-1208, 2001.

[15] F.A. Maruf, U.N. Ogochukwu, P.A. Dim and A.R.A. Alada, "Absence of sex differences in systolic blood pressure and heart rate responses to exercise in healthy young adults," Niger. J. Physiol. Sci, vol. 27(1), pp. 95-100, 2012.

[16] C.L.M. Forjaz, Y. Matsudaira, F.B. Rodrigues, N. Nunes, and C.E. Negrão, "Post-exercise changes in blood pressure, heart rate and rate pressure product at different exercise intensities in normotensive humans," Brazilian J. Med. Biol. Res, vol. 31(10), pp. 1247-1255, 1998.

[17] K. Wielemborek-Musial, K. Szmigielska, J. Leszczynska and A. Jegier, "Blood Pressure Response to Submaximal Exercise Test in Adults," Biomed Res. Int, 2016. 